\title{
Propofol pretreatment attenuates remote kidney injury induced by orthotopic liver autotransplantation, which is correlated with the activation of $\mathrm{Nrf} 2$ in rats
}

\author{
MIAN GE*, GANGJIAN LUO*, WEIFENG YAO*, CHENFANG LUO, \\ SHAOLI ZHOU, DONGDONG YUAN, XINJIN CHI and ZIQING HEI \\ Department of Anesthesiology, The Third Affiliated Hospital, Sun Yat-sen University, \\ Guangzhou, Guangdong 510630, P.R. China
}

Received February 23, 2014; Accepted November 20, 2014

DOI: $10.3892 / \mathrm{mmr} .2014 .3126$

\begin{abstract}
Nuclear factor erythroid 2-related factor 2 (Nrf2) is a critical regulator of the cellular-defense response in protection against oxidative injury. Several studies have demonstrated that propofol ameliorates ischemia/reperfusion injury in a number of organs. However, whether propofol exerts renal protection against liver transplantation via Nrf2 activation remains to be elucidated. The aim of the present study was to investigate the effects of orthotopic liver autotransplantation (OLAT) on renal Nrf2 expression and to determine whether propofol protects against kidney injury induced by OLAT via Nrf2 activation. A total of 24 male Sprague Dawley rats were randomly divided into four groups: sham surgery + normal saline (sham group); OLAT + normal saline (OLAT group); OLAT + propofol $50 \mathrm{mg} / \mathrm{kg}$ (L-Prop group) and OLAT + propofol $100 \mathrm{mg} / \mathrm{kg}$ (H-Prop group). Normal saline and propofol were administered for 3 consecutive days through an intraperitoneal injection prior to surgery. Kidney pathology, blood urea nitrogen (BUN), creatinine $(\mathrm{Cr})$, superoxide anion $\left(\mathrm{O}_{2}{ }^{-}\right)$, hydroxyl radical $(\cdot \mathrm{OH})$, maleic dialdehyde (MDA) and expression levels of Nrf2, Kelch-like ECH-associated protein 1 (Keap1), heme oxygenase-1 (HO-1) and NADP quinine oxidoreductase 1 (NQO1) were assessed $8 \mathrm{~h}$ after OLAT. It was demonstrated that OLAT induced remote kidney damage. Pretreatment with propofol significantly ameliorated renal pathology and abrogated the increase of the $\mathrm{Cr}$ and BUN concentrations, $\mathrm{O}_{2}{ }^{-}$and $\cdot \mathrm{OH}$ activities, and MDA levels induced by OLAT.
\end{abstract}

Correspondence to: Professor Ziqing Hei, Department of Anesthesiology, The Third Affiliated Hospital of Sun Yat-sen University, 660 Tianhe Road, Guangzhou, Guangdong 510630, P.R. China

E-mail: heiziqing@sina.com.cn

${ }^{*}$ Contributed equally

Key words: propofol, kidney injury, orthotopic liver autotransplantation, nuclear factor erythroid 2-related factor 2
In the H-Prop group, Keapl expression in the cytoplasm was decreased and Nrf2 expression in the nucleus was upregulated, accompanied by an increase of HO-1 and NQO1 expression. The present results suggest that propofol pretreatment exerted renal protection against OLAT, with the upregulation of nuclear Nrf2 expression as a potential mechanism.

\section{Introduction}

The incidence of acute kidney injury (AKI) following liver transplantation has been reported to range widely-between $8 \%$ and $78 \%$ (1). Several studies have demonstrated that AKI has an important role in the short-term and long-term survival rate of patients who have received a liver transplant $(2,3)$. The causes and mechanism of AKI induced by liver transplantation are complicated and include intraoperative renal hypoperfusion and the effects of mediators produced endogenously following liver transplantation. Several studies have demonstrated that oxidative stress of the kidney is activated by the aforementioned factors, in addition to being involved in the development of AKI (4-7).

Previously, it has been demonstrated that transcription nuclear factor erythroid 2-related factor 2 (Nrf2), characterized as 'an oxidative stress-sensing guarding regulator', combines with the activating response element and regulates a multitude of cytoprotective genes acting in synergy to remove reactive oxygen species (ROS) through sequential enzymatic reactions, including heme oxygenase-1 (HO-1) and nicotinamide adenine dinucleotide phosphate quinine oxidoreductase1 (NQO1) (7-9). Kelch-like ECH-associated protein 1 (Keap1) is a negative regulator of $\mathrm{Nrf} 2$ and sequesters $\mathrm{Nrf} 2$ in the cytoplasm in its homodimeric isoform, promoting Nrf2 degradation via the ubiquitin-proteasome pathway. An earlier study suggested that upregulation of Nrf2 and Nrf2-dependent phase 2 enzymes assisted to ameliorate kidney ischemic-reperfusion (I/R) injury (10). Nonetheless, the role of the Nrf2-Keap1 system in AKI associated with liver transplantation remains to be elucidated.

Propofol, (2,6)-diisopropylphenol, is a common anesthetic. Propofol has been revealed to ameliorate I/R injury in several organs through potential anti-inflammatory, 
antiapoptotic or antioxidation effects (11-15). Several studies have demonstrated that propofol may enhance the expression of antioxidant enzymes, including superoxide dismutase (SOD) and p47phox $(11,12)$. Nrf2 is the key regulator, which modulates the expression of numerous antioxidant enzymes. However, few studies have been conducted on its effects on Nrf2 expression and its downstream antioxidant and detoxification enzymes, including HO-1 and NQO1. In the present study, a rat orthotopic liver autotransplantation (OLAT) model was used to investigate whether propofol has a protective effect in kidney injury induced by OLAT via Nrf2 activation.

\section{Materials and methods}

Animal and experimental design. A total of 24 male Sprague Dawley (SD) rats (220-280 g) were provided by the Medical Experimental Animal Center of Guangdong Province (Foshan, China). Ethical approval for the present study was provided by the Institutional Animal Care and Use Committee of Sun Yat-Sen University (Guangzhou, China). The animal studies were performed in accordance with the Guide for the Care and Use of Laboratory Animals, issued by the National Institutes of Health (Bethesda, MD, USA).

SD rats were randomly assigned to four groups $(n=6$ in each group). The sham group (sham), which was subjected to abdominal incision, vascular dissection and wound closure without hepatic vascular exclusion and perfusion, and the orthotopic liver autotransplantation group (OLAT) were administered isotonic sodium chloride solution (Sigma-Aldrich, St. Louis, MO, USA) through intraperitoneal (i.p.) injection on each of three consecutive days prior to the experiment. The OLAT + low dose propofol-treated group (L-Prop) received propofol at $50 \mathrm{mg} / \mathrm{kg}$ i.p. for 3 consecutive days prior to OLAT and the OLAT + high dose propofol-treated group (H-Prop) received propofol (Corden Pharma S.P.A, Caponago, Italy) at $100 \mathrm{mg} / \mathrm{kg}$ i.p. for 3 consecutive days prior to OLAT. All rats were sacrificed $8 \mathrm{~h}$ after the sham or OLAT surgeries and kidney tissue was collected for analysis.

Following administration of an ether inhalation anesthetic (Sanpin Chemical Technology, Co., Ltd, Shenzen, China), surgery was performed on the OLAT model, as originally described by Jin et al (16) and modified by Chi et al (17). This model simulated the main surgical steps and pathophysiological course of human liver transplantation, including blocking and unclamping the hepatic artery and portal vein, liver I/R injury, intestinal congestion and hypoxia. The doses of propofol were based on previous experiments in rats, which demonstrated that $50 \mathrm{mg} / \mathrm{kg}$ i.p. of propofol produced sedation and $100 \mathrm{mg} / \mathrm{kg}$ i.p. provided satisfactory anesthesia $(18,19)$.

Histological examination of kidney tissue. The harvested kidneys were fixed in a $10 \%$ formaldehyde solution (Sigma-Aldrich) and embedded in paraffin (Leica Microsystems, Wetzlar, Germany) for histopathological analysis. The kidney paraffin sections $(5 \mu \mathrm{m})$ were stained with hematoxylinandeosin(BeiJingDingGuoChangShengBiotech. Co., Ltd, Beijing, China). The severity of kidney injury was evaluated, in a blinded manner, using a semi-quantitative scale evaluating morphological characteristics of the tubules as suggested by Paller et al (20). Higher scores represented more severe damage (the maximum score per tubule was 10), with points administered for tubular dilatation and tubular epithelial cell flattening (1 point), loss of brush borders (1 point), cell membrane bleb formation (1 or 2 points), interstitial edema (1 point), cellular vacuolization (1 point), cell necrosis (1 or 2 points) and tubular lumen obstruction (1 or 2 points) (21).

Biochemical analysis. Blood urea nitrogen (BUN) and Creatinine $(\mathrm{Cr})$, which were used as renal functional indices, were detected in blood samples with an automatic biochemistry analyzer (Hitachi 7600-020/7170A; Hitachi, Tokyo, Japan).

Tissue preparation for detection of protein content. The collected kidney tissue was frozen at $-80^{\circ} \mathrm{C}$. The frozen kidney tissue was homogenized on ice in 10 volumes of frozen saline using a homogenizer (Polytron; Kinematica, Lucerne, Switzerland). The homogenates were centrifuged for $10 \mathrm{~min}$ at $5,000 \times \mathrm{g}$ and the supernatant was allocated into 6-8 separate tubes and preserved at $-80^{\circ} \mathrm{C}$ until use in biochemical assays. The protein content was determined using a bicinchoninic acid (BCA) protein assay kit (KeyGen Biotech Co., Ltd, Nanjing, China) according to the manufacturer's instructions.

Detection of superoxide anion $\left(\mathrm{O}_{2}{ }^{--}\right)$and hydroxyl radical $(\cdot \mathrm{OH})$ activity in kidney tissue. $\mathrm{O}_{2}{ }^{--}$and $\cdot \mathrm{OH}$, the main oxygen free radicals, were quantified using assay kits (KeyGen Biotech Co., Ltd.) according to the manufacturer's instructions. The quantity of $\mathrm{O}_{2}{ }^{-}$and $\cdot \mathrm{OH}$ in the kidney tissue was expressed in units per milligram of protein (U/mg).

Measurements of the maleic dialdehyde (MDA) content in kidney tissue. The content of MDA was determined as an index of lipid peroxidation, as described by Draper and Hadley (22). The MDA content was assayed using an assay kit (KeyGen Biotech Co., Ltd.) according to the manufacturer's instructions. The kit measures the absorbance at $532 \mathrm{~nm}$ and expresses the content as $\mathrm{nmol} / \mathrm{mg}$ protein.

Western blot analysis. Total protein was extracted from frozen kidney tissue using total protein extraction kits (KeyGen Biotech Co., Ltd.) for NQO1 measurement. Cytosolic and nuclear protein extracts were prepared using nuclear and cytoplasmic protein extraction kits (KeyGen Biotech Co., Ltd.) according to the manufacturer's instructions. The cytoplasmic protein was collected for Keap1, NQO1 and HO-1 measurements, and the nuclear protein was extracted for Nrf2 detection. Protein concentrations were determined using a BCA protein assay reagent kit (KeyGen Biotech Co., Ltd).

Primary antibodies were added to the samples to detect Nrf2 (1:250 dilution, Santa Cruz Biotechnology, Inc., Santa Cruz, CA, USA), Keap1 (1:1,000 dilution, Millipore, Billerica, MA, USA), HO-1 (1:250 dilution, Santa Cruz Biotechnology, Inc.), NQO1 (1:250 dilution, Santa Cruz Biotechnology, Inc.), $\beta$-actin (1:2,000 dilution, Santa Cruz Biotechnology, Inc.) and Lamin B (1:2,000 dilution, Millipore). The relative density of bands was quantified by computerized scanning of the images using AlphaView software version 2.2.14407 (ProteinSimple, Santa Clara, CA, USA) and normalized by $\beta$-actin immunoreactivity to correct for any loading and transfer differences between samples. 

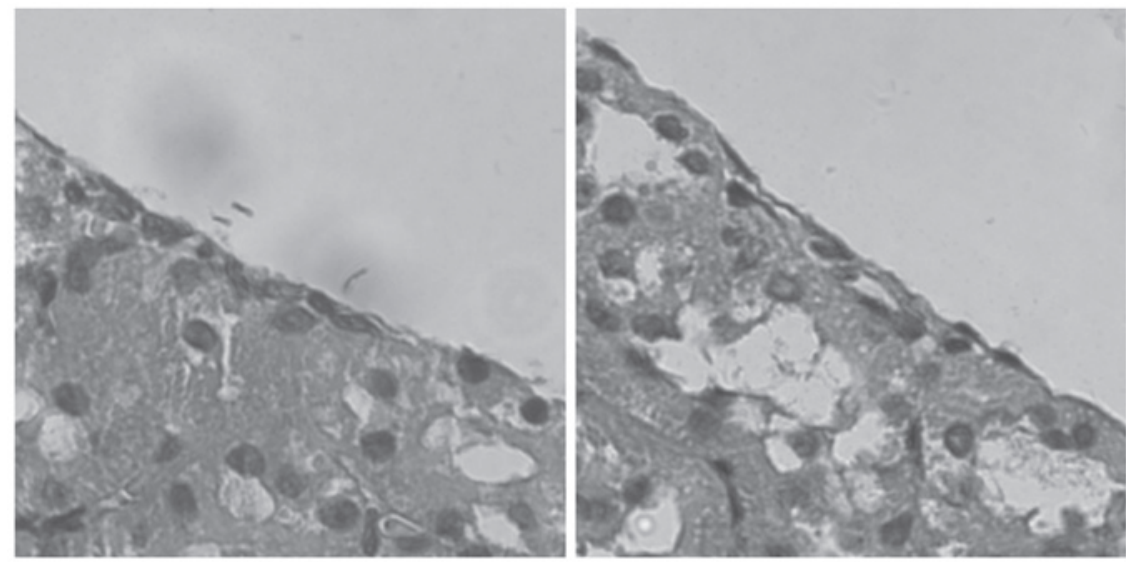

Sham

OLAT
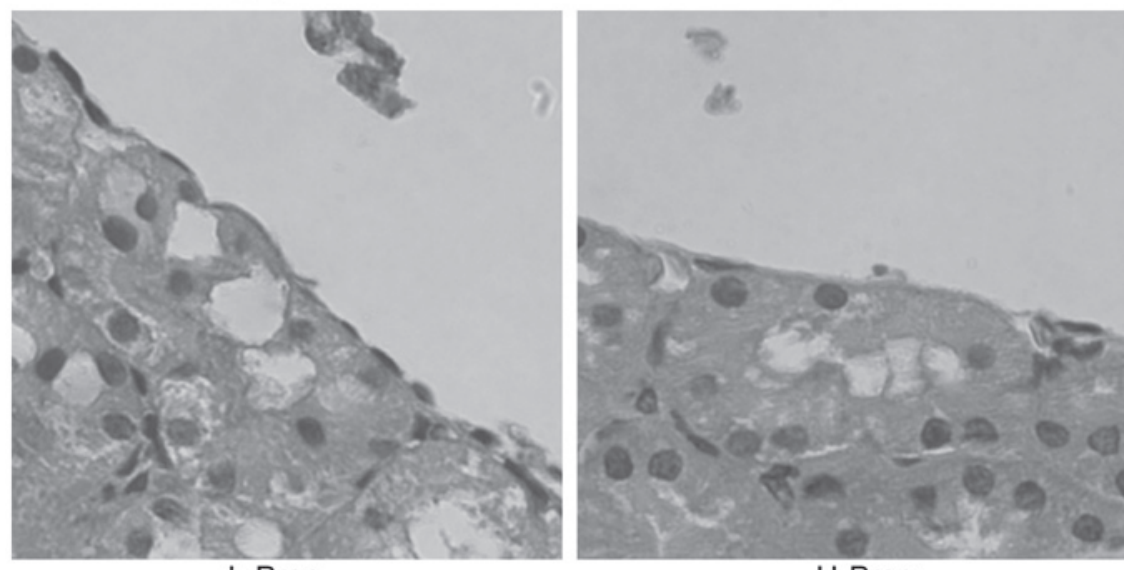

H-Prop

Figure 1. Histopathological analysis of a kidney under microscopy (magnification, x200). L-Prop, the OLAT + low-dose propafol-treated group; H-Prop, the OLAT + high-dose propafol-treated group; OLAT, orthotopic liver autotransplantation.

Immunohistochemical analysis. For immunohistochemical staining, the kidney paraffin sections $(5 \mu \mathrm{m})$ were stained using the immunohistochemistry technique for $\mathrm{Nrf} 2$ as described by Tanaka et al (23). After the sections were washed in phosphate-buffered saline three times, the kidney sections were immersed in $3 \%$ hydrogen peroxide $\left(\mathrm{H}_{2} \mathrm{O}_{2}\right.$; Guangzhou Chemical Reagent Factory, Guangzhou, China) to quench endogenous peroxidase and then blocked in bovine serum Boster Biotech Co., Ltd, Wuhan, China) for $1 \mathrm{~h}$. The kidney sections were incubated with rabbit polyclonal antibody against $\mathrm{Nrf} 2$ (1:50 dilution, Santa Cruz Biotechnology, Inc.) overnight at $4^{\circ} \mathrm{C}$ and then incubated with an anti-rabbit IgG secondary antibody (Santa Cruz Biotechnology, Inc.) and a horseradish-peroxidase-conjugated streptavidin solution. Brown granules in the nucleus indicated positive staining for $\mathrm{Nrf} 2$.

Statistical analysis. Quantitative data, expressed as the mean \pm standard deviation, were statistically analyzed using a one-way analysis of variance for inter-group comparisons using SPSS 17.0 (IBM, Armonk, NY, USA). P<0.05 was considered to indicate a statistically significant difference.

\section{Results}

Histopathological analysis of the kidney using microscopy. As shown in Fig. 1A, there was no detectable change in the sham group, which exhibited normal nuclei and intact brush borders. Significant histological injury of the kidney was observed in the OLAT group (Fig. 1B), including luminal debris formation, tubular cell flattening, cellular vacuolization and loss of brush borders. These effects were attenuated by pretreatment with propofol (Fig. 1C and D), which exhibited less structural damage than that observed in the OLAT group. In the L-Prop group, tubular cell swelling and expansion of renal tubular lumen were observed (Fig. 1C). Conversely, pretreatment with a high dose of propofol markedly ameliorated the renal morphology injury induced by OLAT and resulted in slight tubular dilatation and flattening of the tubular epithelium cells (Fig. 1D).

Serum BUN and Cr levels. Plasma $\mathrm{Cr}$ and BUN are classical indicators, which reflect renal function. OLAT, involving $20 \mathrm{~min}$ of ischemia followed by $8 \mathrm{~h}$ of reperfusion resulted in significant increases in the serum concentration of $\mathrm{Cr}$ and BUN (Fig. 2; $\mathrm{P}<0.05$, versus the sham group). Low- and high-dose propofol pretreatment reduced the increases in serum BUN and $\mathrm{Cr}$ levels $(\mathrm{P}<0.05$, versus the OLAT group).

$\mathrm{O}_{2}{ }^{--}$and $\cdot \mathrm{OH}$ activity in kidney tissue. $\mathrm{O}_{2}{ }^{--}$and $\cdot \mathrm{OH}$ are the main oxygen free radicals. As depicted in Fig. $3 \mathrm{~A}$ and $\mathrm{B}, \mathrm{O}_{2}{ }^{-}$ and $\cdot \mathrm{OH}$ activity markedly increased in the OLAT and L-Prop groups as compared with the sham group $(\mathrm{P}<0.05)$. When a 
A

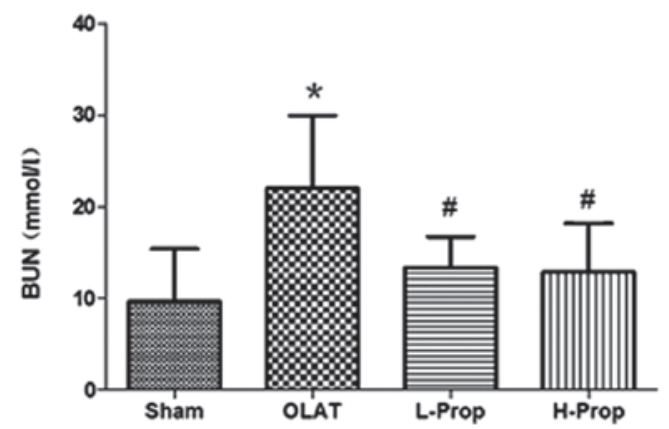

B

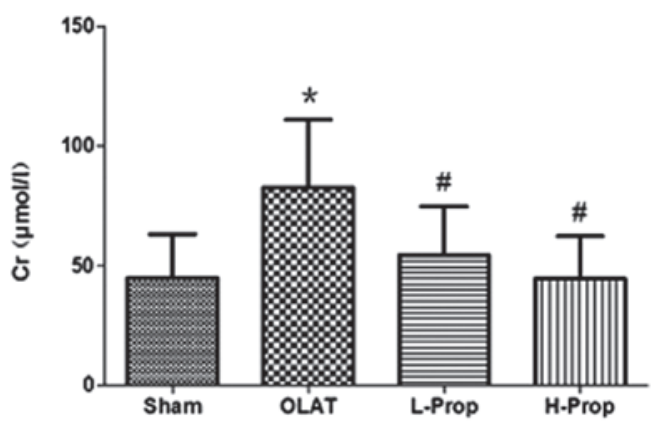

Figure 2. Changes in serum (A) BUN and (B) Cr levels. Results are expressed as the mean \pm standard deviation $(\mathrm{n}=6)$. ${ }^{*} \mathrm{P}<0.05$ compared with the Sham group; ${ }^{\#} \mathrm{P}<0.05$ compared with the OLAT group. L-Prop, the OLAT + low-dose propafol-treated group; H-Prop, the OLAT + high-dose propafol-treated group; OLAT, orthotopic liver autotransplantation; BUN, blood urea nitrogen; $\mathrm{Cr}$, creatinine.

A

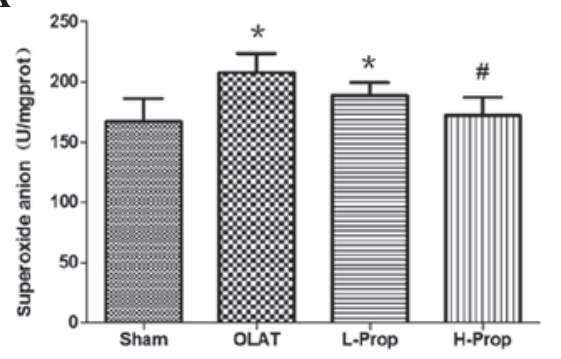

B

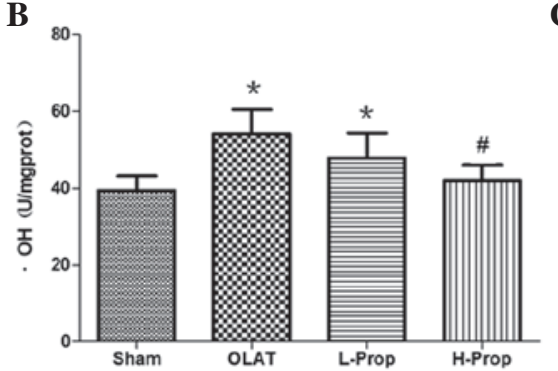

C

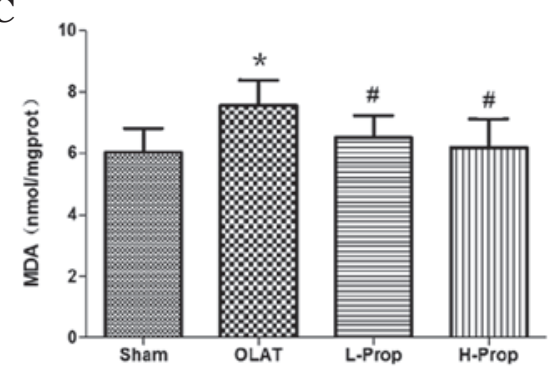

Figure 3. Changes in (A) superoxide anion and (B) $\cdot \mathrm{OH}$ activity and (C) the MDA content in kidney tissue. Results are expressed as the mean \pm standard deviation $(\mathrm{n}=6) .{ }^{*} \mathrm{P}<0.05$ compared with the Sham group; ${ }^{\text {}} \mathrm{P}<0.05$ compared with the OLAT group. L-Prop, the OLAT + low-dose propafol-treated group; H-Prop, the OLAT + high-dose propafol-treated group; OLAT, orthotopic liver autotransplantation; · OH, hydroxyl radical; MDA, maleic dialdehyde.
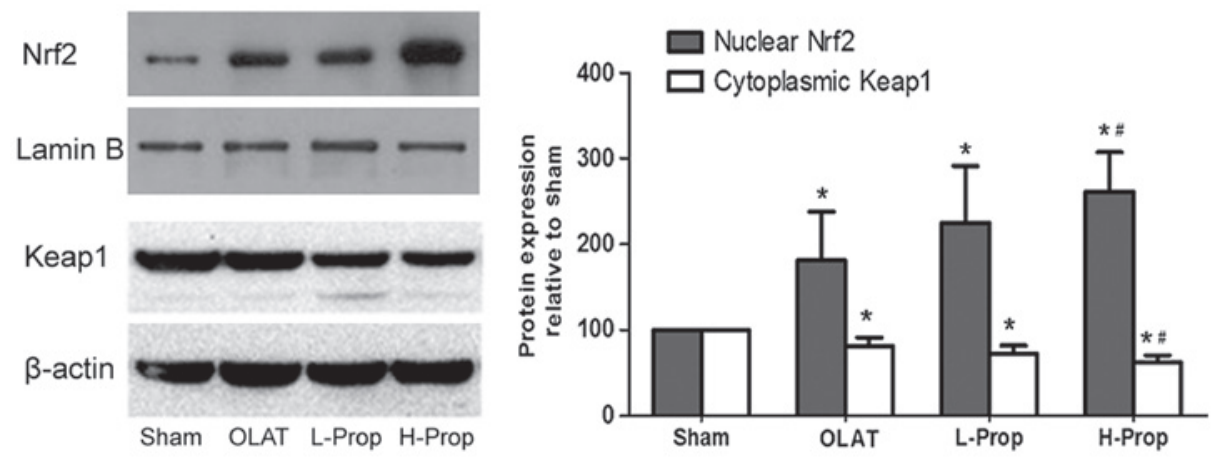

Figure 4. Western blot analysis of expression of Nrf2 in the nucleus and Keap1 in the cytoplasm of the kidney tissue. Results are expressed as the mean \pm standard deviation $(\mathrm{n}=6) .{ }^{*} \mathrm{P}<0.05$ compared with the Sham group; ${ }^{\prime} \mathrm{P}<0.05$ compared with the OLAT group. L-Prop, the OLAT + low-dose propafol-treated group; H-Prop, the OLAT + high-dose propafol-treated group; OLAT, orthotopic liver autotransplantation; Nrf2, nuclear factor erythroid 2-related factor 2; Keap1, Kelch-like ECH-associated protein 1.

high dose of propofol $(100 \mathrm{mg} / \mathrm{kg}$ i.p. $)$ was administered, the increases in $\mathrm{O}_{2}{ }^{-}$and $\cdot \mathrm{OH}$ activity were significantly suppressed $(\mathrm{P}<0.05$, versus the OLAT group). Low-dose propofol pretreatment reduced the increases in $\mathrm{O}_{2}^{-}$-and $\cdot \mathrm{OH}$ activity, however no significant difference was observed $(P>0.05$, versus the OLAT group; Fig. 3A and B).

MDA content in kidney tissue. MDA is the product of lipid peroxidation damage. The MDA content in the kidney was significantly higher in the OLAT group than in the sham group ( $\mathrm{P}<0.05$, versus the sham group). However, this increase was significantly reduced by pretreatment with propofol $(\mathrm{P}=0.04$,
L-Prop group, versus the OLAT group; $\mathrm{P}=0.009$, H-Prop group, versus the OLAT group; Fig. 3C).

Expression of Nrf2 and Keapl in kidney tissue. Nrf2 is an unstable protein with a short half-life $(<20 \mathrm{~min})$ and is dependent on the status of the Keap1/Nrf2 system. Keap1, which sequesters Nrf2 in the cytoplasm and prevents it from translocation to the nucleus, is crucial in the regulation of the Nrf2 pathway (7-9). As shown in Fig. 4, there was low expression of Nrf2 in the nucleus in the sham group. By contrast, expression of the Keap1 protein was very high in the cytoplasm in this group. Compared with the sham group, 


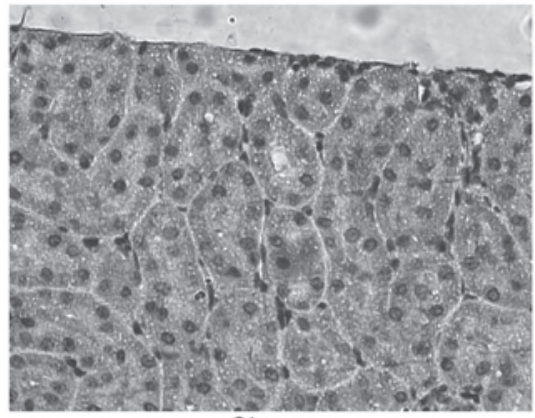

Sham

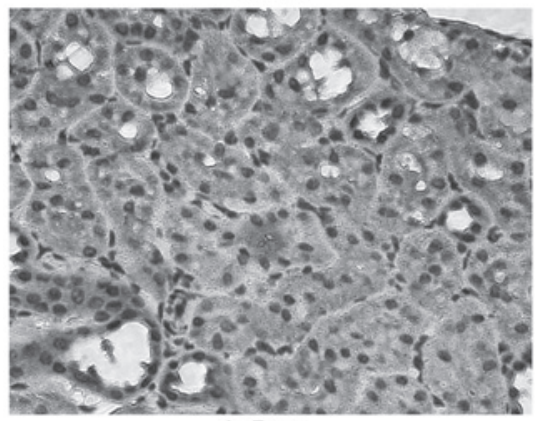

L-Prop

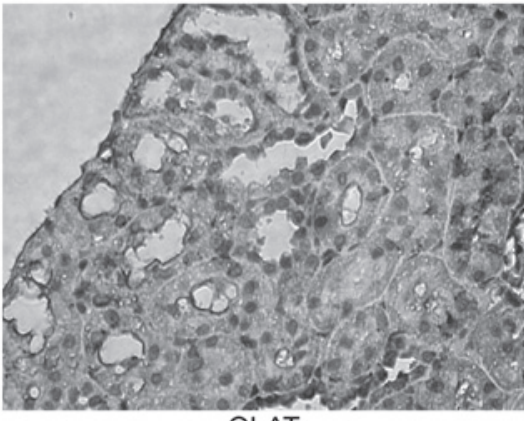

OLAT

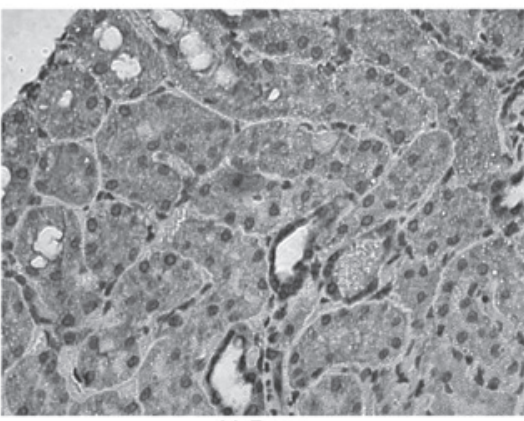

H-Prop

Figure 5. Immunohistochemical analysis of expression of nuclear factor erythroid 2-related factor 2 in kidney (magnification $x 400$ ). L-Prop, the OLAT + low-dose propafol-treated group; H-Prop, the OLAT + high-dose propafol-treated group; OLAT, orthotopic liver autotransplantation.

A
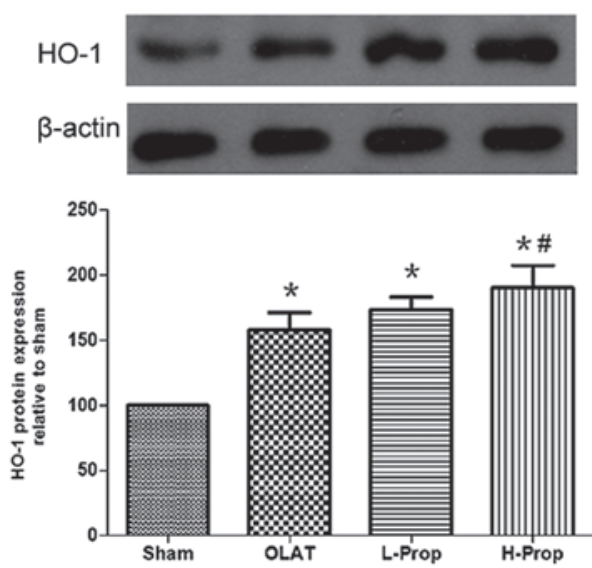

B

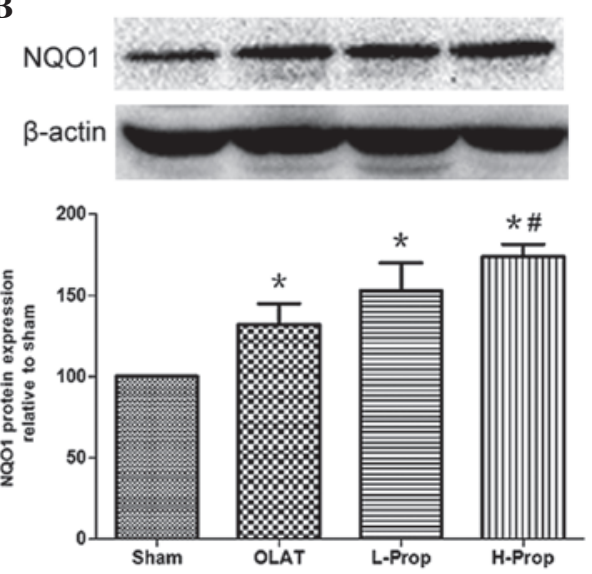

Figure 6. Western blot analysis of the expression of (A) HO-1 and (B) NQO1 in renal tissue. Results are expressed as the mean \pm standard deviation (n=6). "P<0.05 compared with the Sham group; ${ }^{~} \mathrm{P}<0.05$ compared with the OLAT group; L-Prop, the OLAT + low-dose propafol-treated group; H-Prop, the OLAT + high-dose propafol-treated group; OLAT, orthotopic liver autotransplantation; HO-1, heme oxygenase-1; NQO1, NADP quinine oxidoreductase 1.

the OLAT, L-Prop and H-Prop groups exhibited a significant increase in expression of Nrf2 in the nucleus and an evident decrease in expression of Keap1 in the cytoplasm $(\mathrm{P}<0.05$, versus the sham group). Furthermore, the increase of nuclear Nrf2 expression and the decrease of cytoplasm Keap1 expression were more marked in the H-Prop group than those in the OLAT group $(\mathrm{P}<0.05$, versus the OLAT group), indicating that the high-dose propofol pretreatment inhibited Keap1 expression in the cytoplasm and then promoted Nrf2 to translocate into nucleus.

Consistent with the western blot analysis, the expression of Nrf2 was minimal, as demonstrated by the lack of clear visualization of its immunoreactivity in the sham group. Compared with the sham group, the proportion of Nrf2-positive cells with brown staining in the nuclei was significantly higher in the OLAT, L-Prop and H-Prop groups. Furthermore, compared with the OLAT group, positive expression of Nrf2 was markedly increased in the H-Prop group (Fig. 5).

Expression of HO-1 and NQO1 in renal tissue. HO-1 and NQO1 are two typical downstream phase II antioxidant enzymes mediated by Nrf2 (7-9). Expression of HO-1 and NQO1 was markedly increased in the OLAT, L-Prop and H-Prop groups ( $\mathrm{P}<0.05$, versus the sham group), whereas the HO-1 and NQO1 proteins were expressed at low levels in the sham group. However, pretreatment with high-dose propofol significantly augmented the expression of HO-1 and NQO1 compared with the OLAT group $(\mathrm{P}<0.05$, versus the OLAT group; Fig. 6). 


\section{Discussion}

OLAT is a well-established liver transplantation model, including a superior vena cava, inferior vena cava and hepatic portal vein block, hepatic I/R and cold liver preserving fluid perfusion. The present study provided evidence that liver transplantation induces remote kidney damage, which contributes to the progression of oxidative damage. Pretreatment with propofol, a widely used anesthetic, significantly ameliorated renal dysfunction and pathology injury induced by OLAT. Furthermore, propofol inhibited the expression of Keapl in the cytoplasm, upregulated the expression of $\mathrm{Nrf} 2$ in the nucleus and increased HO1 and NQO1 expression. Propofol also reduced OLAT-induced increases in $\mathrm{O}_{2}{ }^{--}$and $\cdot \mathrm{OH}$ activity as well as the MDA content. These results suggest that propofol's renal protective effects against OLAT may be partly due to activation of the Keap1/Nrf2 pathway.

It has been established that remote kidney injury is frequently induced by $\mathrm{I} / \mathrm{R}$ injury during liver transplantation $(3,24)$. Previous studies revealed that $\mathrm{I} / \mathrm{R}$ injury resulted mainly in the production of ROS, which are formed during reperfusion (4-7). These ROS, including hydroxyl radicals $(\cdot \mathrm{OH})$ and superoxide anions $\left(\mathrm{O}_{2}^{--}\right)$, are key mediators of renal reperfusion injury (25) and cause lipid peroxidation of the renal cell membrane, which results in intracellular calcium overload and subsequent necrotic cell death $(26,27)$. In the present study, a significant increase in renal $\mathrm{O}_{2}{ }^{--}, \cdot \mathrm{OH}$ activity and MDA levels was observed, indicating that liver transplantation induced considerable ROS generation and lipid peroxidation damage to the kidney. Kadkhodaee et al (4) reported that $90 \mathrm{~min}$ of liver ischemia and $4 \mathrm{~h}$ of reperfusion caused an increase in kidney MDA levels and a decrease in catalase activities and SOD. The results of the study of Kadkhodaee et al and the present study provided evidence of remote kidney injury induced by liver transplantation.

$\mathrm{Nrf2}$ is a critical regulator of the cellular defense response to protect against oxidative injury $(10,28-34)$. Under conditions of oxidative stress or in the presence of an inducer, $\mathrm{Nrf} 2$ translocates to the nucleus to activate transcription of Nrf2-regulated genes and upregulate antioxidant enzymes and phase II detoxification enzymes, including HO-1 and NQO1. Recent studies (32) have demonstrated that hepatic I/R may lead to kidney dysfunction, but renal Nrf2 activation and the subsequent upregulation of HO-1 and NQO1 contributes to the recovery of renal function. Liu et al (33) observed that Nrf2-deficiency worsens renal function, histology and survival rate as well as enhancing susceptibility to ischemic and nephrotoxic acute kidney injury, indicating a cytoprotective effect of Nrf2, which functions against oxidative stress. Similar to these studies, in the present study, it was observed that expression of Nrf2 in the nucleus was modestly increased, whereas Keap1 expression in the cytoplasm was decreased at $8 \mathrm{~h}$ after OLAT, accompanied by the rise of expression of $\mathrm{HO} 1$ and NQO1, suggesting a defensive mechanism to protect the kidney from organ dysfunction induced by OLAT. However, the degree of renal morphological injury and the increase of serum $\mathrm{Cr}$ and BUN concentrations were marked, implying that the increases in antioxidant enzymes were not sufficient to inhibit oxidative damage at this time and suggesting that specific drugs are required to be used to enhance the expres- sion of Nrf2 and its downstream antioxidant enzymes as well as strengthen the defense response.

Several studies have demonstrated that propofol ameliorates I/R injury in several organs, including the heart, liver, kidney and brain, through improvement of antioxidant enzyme activity (11-15). The association between propofol and HO-1, a type of Nrf2 downstream regulation antioxidant enzyme, has been confirmed. Xu et al (35) demonstrated that propofol may protect cardiomyocytes against $\mathrm{H}_{2} \mathrm{O}_{2}$-mediated cytotoxicity through increased expression of HO-1. Wang et al (36), suggested that propofol may improve renal I/R injury in rats partly through the induction of HO-1 expression and revealed that propofol may induce antioxidant effects via HO-1 activation. However, whether Nrf 2 is important in propofol-mediated protection remains to be elucidated. In the current study, sedation and anesthesia doses were selected to demonstrate that propofol pretreatment prevents OLAT-induced pathological and functional injury to the kidney. Compared with the model group, propofol, particularly in the high dose group, significantly inhibited Keap1 expression, increased Nrf2 nuclear translocation, promoted the expression levels of HO-1 and NQO1, and abrogated the increase of $\mathrm{O}_{2}{ }^{--}$and $\cdot \mathrm{OH}$ activity, as well as MDA levels, reducing the oxidative damage in the kidney induced by OLAT.

In conclusion, propofol pretreatment exerted a renal protective effect against OLAT. The potential mechanism for this effect is through upregulation of nuclear Nrf2 expression.

\section{Acknowledgements}

Financial support and sponsorship: The present study was in part supported by the National Natural Science Foundation of China (grant nos. 81170449 and 81372090).

\section{References}

1. Saner FH, Cicinnati VR, Sotiropoulos G, et al: Strategies to prevent or reduce acute and chronic kidney injury in liver transplantation. Liver Int 32: 179-188, 2013.

2. Narayanan Menon KV, Nyberg SL, Harmsen WS, et al: MELD and other factors associated with survival after liver transplantation. Am J Transplant 4: 819-825, 2004.

3. Gainza FJ, Valdivieso A, Quintanilla N, et al: Evaluation of acute renal failure in the liver transplantation perioperative period: incidence and impact. Transplant Proc 34: 250-251, 2002.

4. Kadkhodaee M, Mikaeili S, Zahmatkesh M, et al: Alteration of renal functional, oxidative stress and inflammatory indices following hepatic ischemia-reperfusion. Gen Physiol Biophys 31: 195-202, 2012.

5. Heyman SN, Rosen S and Rosenberger C: A role for oxidative stress. Contrib Nephrol 174: 138-148, 2011.

6. Wu QQ, Wang Y, Senitko M, et al: Bardoxolone methyl (BARD) ameliorates ischemic AKI and increases expression of protective genes Nrf2, PPAR $\gamma$, and HO-1. Am J Physiol Renal Physiol 300: F1180-F1192, 2011.

7. Saito H: Toxico-pharmacological perspective of the Nrf2-Keap1 defense system against oxidative stress in kidney diseases. Biochem Pharmacol 85: 865-872, 2013.

8. Copple IM: The Keap1-Nrf2 cell defense pathway - a promising therapeutic target? Adv Pharmacol 63: 43-79, 2012.

9. Magesh S, Chen Y and Hu L: Small molecule modulators of Keap1-Nrf2-ARE pathway as potential preventive and therapeutic agents. Med Res Rev 32: 687-726, 2012.

10. Yoon HY, Kang NI, Lee HK, et al: Sulforaphane protects kidneys against ischemia-reperfusion injury through induction of the Nrf2-dependent phase 2 enzyme. Biochem Pharmacol 75: 2214-2223, 2008. 
11. Tsai YC, Huang CC, Chu LM and Liu YC: Differential influence of propofol on different cell types in terms of the expression of various oxidative stress-related enzymes in an experimental endotoxemia model. Acta Anaesthesiol Taiwan 50: 159-166, 2012

12. Yang S, Chou WP and Pei L: Effects of propofol on renal ischemia/reperfusion injury in rats. Exp Ther Med 6: $1177-1183,2013$.

13. Yuzbasioglu MF, Aykas A, Kurutas EB, et al: Protective effects of propofol against ischemia/reperfusion injury in rat kidneys. Ren Fail 32: 578-583, 2010.

14. Popic J, Pesic V, Milanovic D, et al: Propofol-induced changes in neurotrophic signaling in the developing nervous system in vivo. PLoS One 7: e34396, 2012.

15. LiH,TanJ,ZouZ,Huang CG and ShiXY:Propofol post-conditioning protects against cardiomyocyte apoptosis in hypoxia/reoxygenation injury by suppressing nuclear factor-kappaB translocation via extracellular signal-regulated kinase mitogen-activated protein kinase pathway. Eur J Anaesthesiol 28: 525-534, 2011.

16. Jin $\mathrm{C}$, Zhang PJ, Wu XM, et al: Impact of hypoxic preconditioning on apoptosis and its possible mechanism in orthotopic liver autotransplantation in rats. Hepatobiliary Pancreat Dis Int 8: 40-45, 2009.

17. Chi X, Zhang A, Luo G, et al: Knockdown of myeloid differentiation protein-2 reduces acute lung injury following orthotopic autologous liver transplantation in a rat model. Pulm Pharmacol Ther 26: 380-387, 2013.

18. Liu KX, Chen SQ, Huang WQ, et al: Propofol pretreatment reduces ceramide production and attenuates intestinal mucosal apoptosis induced by intestinal ischemia/reperfusion in rats. Anesth Analg 107: 1884-1891, 2008.

19. Mu X, Wu A, Wu J, et al: Effects of anesthetic propofol on release of amino acids from the spinal cord during visceral pain. Neurosei Lett 484: 206-209, 2010

20. Paller MS, Hoidal JR and Ferris TF: Oxygen free radicals in ischemic acute renal failure in the rat. J Clin Inves 74 1156-1164, 1984.

21. Wang HH, Zhou HY, Chen CC, Zhang XL and Cheng G: Propofol attenuation of renal ischemia/reperfusion injury involves heme oxygenase-1. Acta Pharmacol Sin 28: 1175-1180, 2007.

22. Draper HH and Hadley M: Malondialdehyde determination as index of lipid peroxidation. Methods Enzymol 186: 421-431, 1990.

23. Tanaka N, Ikeda Y, Ohta Y, et al: Expression of Keap1-Nrf2 system and antioxidative proteins in mouse brain after transient middle cerebral artery occlusion. Brain Res 25: 246-253, 2011.

24. Karapanagiotou A, Kydona C, Dimitriadis C, et al: Acute kidney injury after orthotopic liver transplantation. Transplant Proc 44: 2727-2729, 2012
25. Paller MS and Neumann TV: Reactive oxygen species and rat renal epithelial cells during hypoxia and reoxygenation. Kidney Int 40: 1041-1049, 1991.

26. Salahudeen AK: Role of lipid peroxidation in $\mathrm{H}_{2} \mathrm{O}_{2}$ induced renal epithelial (LLCPK1) cell injury. Am J Physiol 268 F30-F38, 1995.

27. Sheridan AM, Fitzpatrick S, Wang C, Wheeler DC and Lieberthal W: Lipid peroxidation contributes to hydrogen peroxide induced cytotoxicity in renal epithelial cells. Kidney Int 49: 88-93, 1996.

28. Leonard MO, Kieran NE,Howell K, etal: Reoxygenation-specific activation of the antioxidant transcription factor Nrf 2 mediates cytoprotective gene expression in ischemia-reperfusion injury. FASEB J 20: 2624-2626, 2006.

29. Sun Q, Meng QT, Jiang Y, et al: Ginsenoside Rb1 attenuates intestinal ischemia reperfusion induced renal injury by activating Nrf2/ARE pathway. Molecules 17: 7195-7205, 2012.

30. Zhang X, Xiao Z, Yao J, et al: Participation of protein kinase $C$ in the activation of Nrf2 signaling by ischemic preconditioning in the isolated rabbit heart. Mol Cell Biochem 372: 169-179, 2013.

31. Li M, Zhang X, Cui L, et al: The neuroprotection of oxymatrine in cerebral ischemia/reperfusion is related to nuclear factor erythroid 2-related factor 2 (nrf2)-mediated antioxidant response: role of nrf 2 and hemeoxygenase- 1 expression. Biol Pharm Bull 34: 595-601, 2011.

32. Tanaka Y, Maher JM, Chen C, et al: Hepatic ischemia-reperfusion induces renal heme oxygenase-1 via NF-E2-related factor 2 in rats and mice. Mol Pharmacol 71: 817-825, 2007.

33. Liu M, Grigoryev DN, Crow MT, et al: Transcription factor Nrf2 is protective during ischemic and nephrotoxic acute kidney injury in mice. Kidney Int 76: 277-285, 2009.

34. Lee YM, Shin JW, Lee EH, et al: Protective effects of propofol against hydrogen peroxide-induced oxidative stress in human kidney proximal tubular cells. Korean J Anesthesiol 63: 441-446, 2012

35. Xu JJ and Wang YL: Propofol attenuation of hydrogen peroxide-mediated oxidative stress and apoptosis in cultured cardiomyocytes involves heme oxygenase-1. Eur J Anaesth 25: 395-402, 2008

36. Wang HH, Zhou HY, Chen CC, et al: Propofol attenuation of renal ischemia/reperfusion injury involves heme oxygenase-1. Acta Pharmacol Sin 28: 1175-1180, 2007. 